\title{
Effet de Albizia lebbeck (L.) Benth. et Gliricidia sepium (Jacq.) Kunth ex Walp. sur les paramètres de fertilité du sol
}

KABORE W. Bruno ${ }^{1}$; SOULAMA Soungalo ${ }^{2 *}$, BAMBARA Dasmané2; BEMBAMBA Michel'1; HIEN Edmond 1

1 Laboratoire de Sol et Environnement, Université Joseph KI-Zerbo, 03 BP 7021 Ouagadougou 03, Burkina Faso.

2Département Environnement et Forêts, INERA, CNRST 03 BP 476 Ouagadougou 03, Burkina Faso

*Auteur correspondant: SOULAMA Soungalo, soulsoung@yahoo.fr;

Original submitted in on 23rd October 2020. Published online at www.m.elewa.org/journals/ on 31st December 2020 https://doi.org/10.35759/JABs.156.2

\section{RESUME}

Objectif : L'agroforesterie est l'une des techniques permettant d'atténuer les effets néfastes de la dégradation des terres. Cette étude vise à évaluer le potentiel agroforestier de deux espèces de légumineuses Albizia lebbeck (L.) Benth. et Gliricidia sepium (Jacq.) Kunth ex Walp., promues dans l'agroforesterie.

Méthodologie et résultats: Pour cela les paramètres physico-chimiques et biologiques des sols sous houppier et hors houppier de Albizia lebbeck et Gliricidia sepium ont été caractérisés. Des tests de comparaison de moyennes, une ANOVA à mesures répétées et une analyse en composante principale (ACP) ont permis de comparer les sols sous houppier et hors houppier des deux espèces. Les résultats montrent que Albizia lebbeck et Gliricidia sepium ont eu un effet positif sur la biomasse microbienne, l'activité respiratoire et la densité de spores des sols $(P<0,05)$. Albizia lebbeck et Gliricidia sepium ont également induit une amélioration des paramètres physico-chimiques des sols sous houppiers.

Conclusion et application des résultats : Ces résultats permettent de conclure que l'utilisation des deux espèces de ligneuses en association avec les cultures garantit une exploitation durable des sols. Ces résultats peuvent servir à améliorer la production végétale en agroforesterie.

Mots clés : Fertilité des sols, agroforesterie, Albizia lebbeck ; Gliricidia sepium; Burkina Faso

\begin{abstract}
Effect of Albizia lebbeck (L.) Benth. and Gliricidia sepium (Jacq.) Kunth ex Walp. on soil fertility parameters

Objective: Agroforestry is one of the techniques to mitigate the negative effects of land degradation. This study aims to assess the agroforestry potential of two species of legumes Albizia lebbeck (L.) Benth. and Gliricidia sepium (Jacq.) Kunth ex Walp., promoted in agroforestry.

Methodology and Results: For this, the physico-chemical and biological parameters of the soils under the crown and outside the crown of Albizia lebbeck and Gliricidia sepium were characterized. Comparison of means tests, repeated measures ANOVA and principal component analysis (PCA) were used to compare the soils under crown and outside the crown of the two species. The results show that Albizia lebbeck and Gliricidia sepium had a positive effect on microbial biomass, respiratory activity and soil spores' density $(P$
\end{abstract}


<0.05). Albizia lebbeck and Gliricidia sepium also induced an improvement in the physico-chemical parameters of soils under crowns.

Conclusion and application of results :These results allow to conclude that the use of the two woody species in association with the cultures guarantees a sustainable use of soils. These results can be used to improve crop production in agroforestry.

Keywords: soil fertility, agroforestry, Albizia lebbeck; Gliricidia sepium; Burkina Faso

\section{INTRODUCTION}

Durant ces dernières décennies les risques de dégradation des terres constituent l'une des préoccupations majeures des environnementalistes. Les mauvaises pratiques agricoles (défrichement, utilisation anarchique des produits agro-chimiques, ) constituent des facteurs importants de la dégradation des terres agricoles (Savadogo et al., 2017 ; Bambara et al., 2018 ; Yaméogo et al., 2019). Les conditions climatiques jumelées aux facteurs anthropiques exposent les sols à l'érosion, détruisant ainsi sa structure (Zongo, 2013 ; Tiendrébéogo et al., 2019 ; Ouédraogo et al., 2019). Le taux de matière organique, les teneurs en éléments nutritifs facilement assimilables ( $\mathrm{N}$ - P- K), le pouvoir tampon du sol sont en baisse (Coulibaly et al., 2012; REEB IV, 2017). Du point de vu biologique on note la perte d'habitats, la perte des espèces naturelles et la perte des macro et microorganismes du sol (Ouédraogo et al., 2014 ; NDT, 2017). La restauration des écosystèmes dégradés constitue donc un enjeu en termes de préservation de la biodiversité et des ressources naturelles (Kouadio et al., 2014). Les travaux

\section{MATERIEL ET METHODES}

Zone d'étude : Le site d'étude (Figure 1) est situé dans la localité de Cassou, commune de Cassou, province du Ziro, Région du Centre-ouest du Burkina Faso. Les coordonnées géographiques du site (en UTM zone 30N) sont : Longitude : 0609087 ; Latitude : 1279766 ; Altitude $: 335 \mathrm{~m}$. La province du Ziro où se situe le site d'étude, est couverte par un climat de type Nord soudanien. La pluviométrie annuelle est comprise entre 800 et $900 \mathrm{~mm}$ (BUNASOLS, 2001). Le couvert végétal de la province du Ziro est constitué de savanes arborées à arbustives, effectués sur la restauration des sols révèlent l'importance de la matière organique dans la récupération des terres dégradés (Nacro et al., 2010; Somé et al., 2015). Les ligneux du groupe des légumineuses sont des arbres dont l'activité enrichit la couche arable du sol (Gnahoua et al., 2013). L'association cultures-légumineuses dans les exploitations agricoles permet de rétablir la fertilité et d'exploiter durablement les sols (Ndiaye et al., 2012; Villenave et al., 2018). En effet les litières des ligneux par décomposition naturelle constituent une source importante de matières organiques pour les sols. L'utilisation des ligneux au champ impliquent donc la détermination de leurs potentiels agroforestiers. D'où notre intérêt à tester l'effet de deux espèces de légumineuses Albizia lebbeck et Gliricidia sepium sur les paramètres physicochimiques et biologiques du sol. L'objectif de cette étude d'évaluer l'effet des espèces Albizia lebbeck et Gliricidia sepium sur la fertilité du sol. Notre hypothèse est que les espèces Albizia lebbeck et Gliricidia sepium améliorent les propriétés physicochimiques et biologiques du sol.

de savanes boisées, et de formations ripicoles. Les sols de la classe des sols à sesquioxydes de fer et de manganèse sont les plus rencontrés dans la province du Ziro. Ces sols appartiennent au groupe des sols ferrugineux tropicaux lessivés caractérisés par un indice de lessivage inférieur à 1/1,4 (BUNASOLS, 2001). L'agriculture reste la principale activité humaine dans la province du Ziro. Le site est constitué d'un agrosystème dans lequel deux espèces de légumineuses ligneuses sont utilisées en association avec les cultures annuelles. 
Kabore et al., J. Appl. Biosci. 2020 Effet de Albizia lebbeck (L.) Benth. et Gliricidia sepium (Jacq.) Kunth ex Walp. sur les paramètres de fertilité du sol

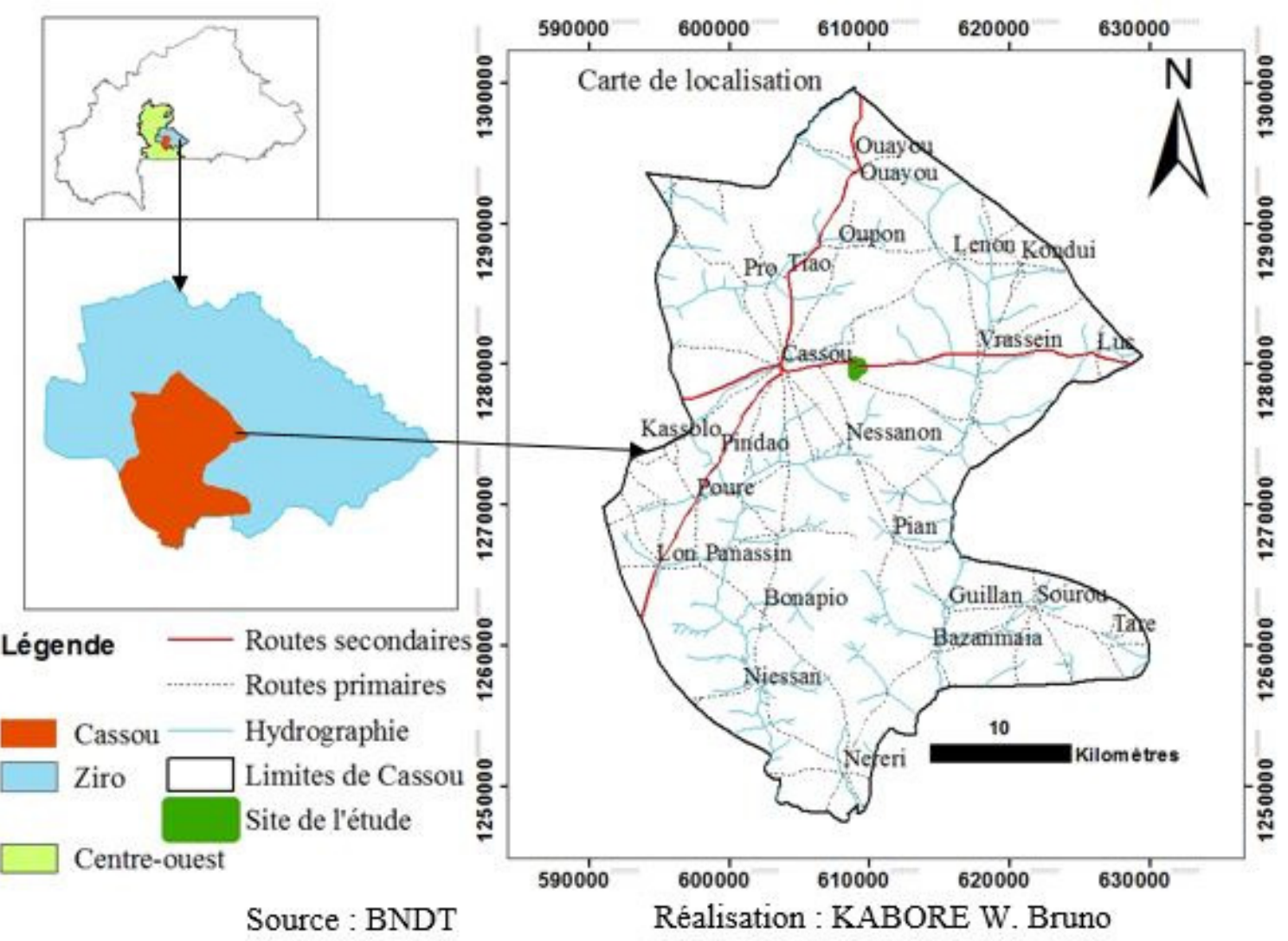

Figure 1 : Zone d'étude

Collecte des données: Deux espèces d'arbres fertilitaires ont été considérées dans l'échantillonnage à cause de leur dominance dans le site. II s'agit de : Gliricidia sepium et Albizia lebbeck (photos).

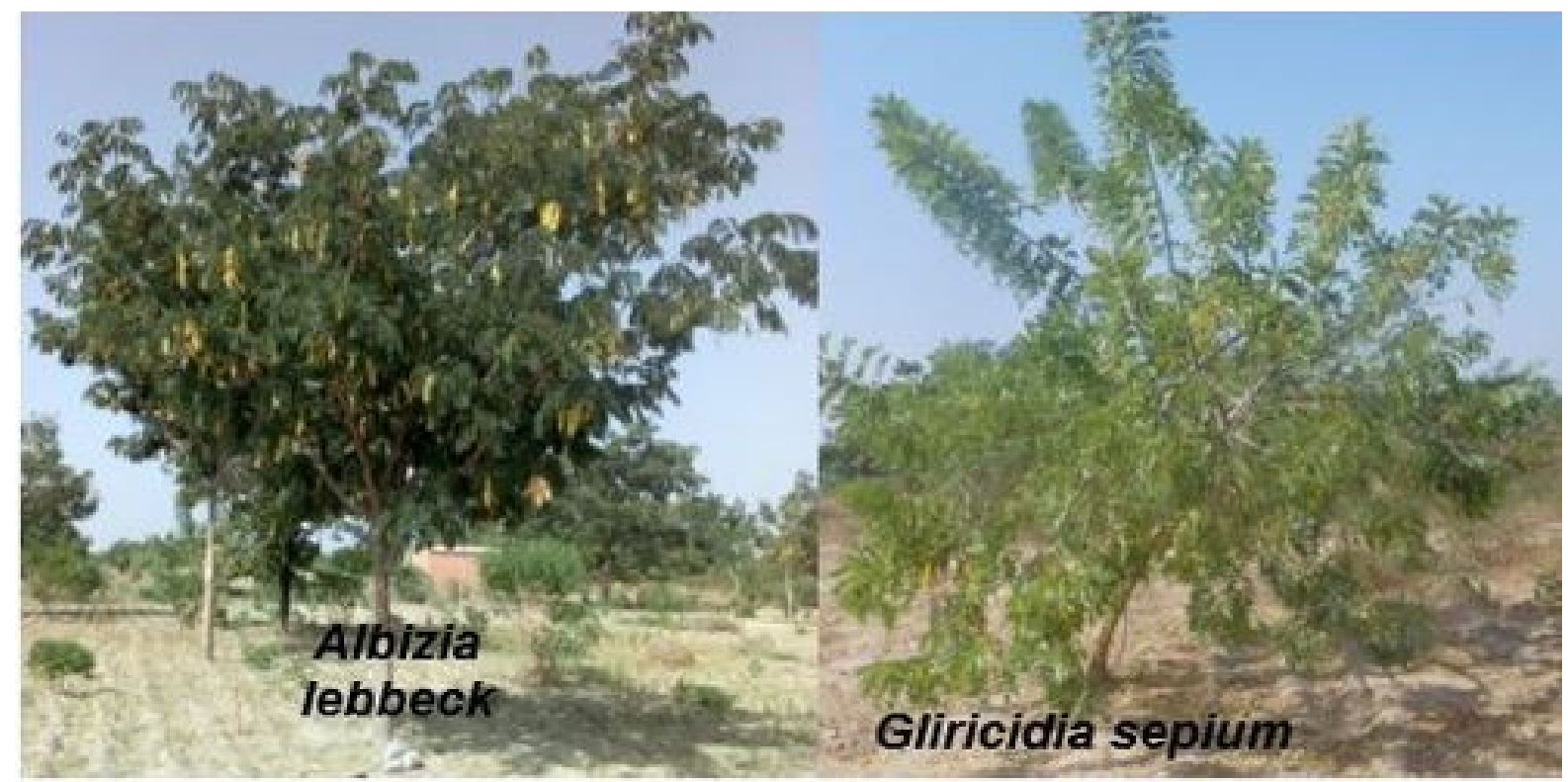

Photo 1 : Gliricidia sepium et Albizia lebbeck 
Pour chaque espèce, quatre pieds ont été choisis en respectant un écart d'au moins $10 \mathrm{~m}$ avec les autres pieds d'arbres. Cinq (05) prélèvements de sol ont été effectués sous le houppier des pieds choisis. Sous chaque houppier quatre prélèvements sont faits aux quatre points cardinaux (est, ouest, nord et sud) à l'intérieur du demi $(1 / 2)$ rayon du houppier et dans l'horizon 0-30 cm à l'aide d'une tarière (Yélémou et al., 2013 ; Fanta, 2016). Ces quatre prélèvements sont mélangés pour former un échantillon composite duquel sont prélevés les 04 échantillons. Quatre autres échantillons témoins sont pris similairement dans des zones éloignées d'au moins $10 \mathrm{~m}$ des arbres fertilitaires, à l'intérieur du champ (Figure 2). Les échantillons ont été ensuite séchés à l'ombre, tamisés à $2 \mathrm{~mm}$ et conservés à température ambiante avant les analyses au laboratoire.

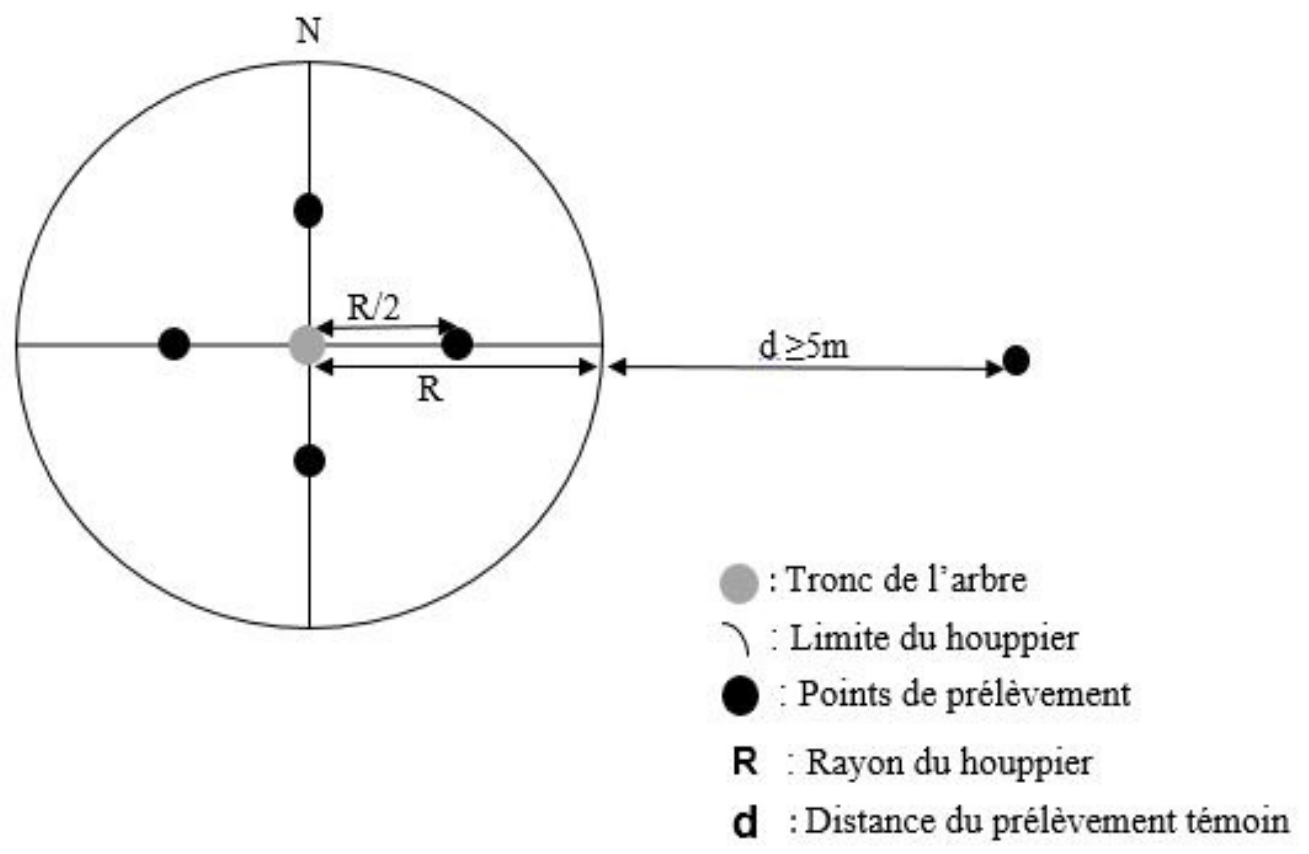

Figure 2 : Schéma de l'échantillonnage du sol

Analyses physico-chimiques : La texture du sol, le carbone organique, l'azote total, le potassium total, le phosphore assimilable, le potentiel hydrogène $(\mathrm{PH})$ ont été analysés. La texture du sol a été déterminée par la méthode internationale à la pipette Robinson (Robinson, 1992). Les fractions distinguées sont : l'argile, les limons fins, les limons grossiers, les sables fins et les sables grossiers. Le carbone organique a été déterminé par la méthode de Walkley et Black (1934). L'azote total a été déterminé par la méthode de Kjeldahl (Bremner, 1965). Le potassium total a été déterminé à l'aide d'un photomètre à flamme (Skinner, 1967). Le phosphore assimilable a été dosé à l'auto analyseur automatique selon la méthode Bray-I (Bray et Kurtz, 1945). Le pHe a été mesuré en utilisant directement une électrode électronique dans une suspension de sol dilué à l'eau distillée $(1 / 2,5)$.
Analyses biologiques: La biomasse microbienne, l'activité respiratoire et les spores du sol ont été évaluées. La biomasse microbienne a été déterminée selon la méthode fumigation-incubation de Jenkinson et Powlson (1976). L'activité respiratoire du sol est mesurée par la méthode du test respirométrique, par piégeage du $\mathrm{CO}_{2}$ dans la soude et titrage par l'acide chlorhydrique (Dommergue, 1960). L'extraction des spores est faite par la méthode du tamisage humide de Gerdemann et Nicolson (1963).

Analyses statistiques: Une Analyse en Composante Principale (ACP) a été faite avec le logiciel XLSTAT 7.5.2 afin de regrouper les différentes entités physicochimiques et biologiques suivant les espèces étudiées et le témoin. Des analyses de variance (ANOVA) et des tests de comparaison des moyennes des paramètres biologiques ont été réalisées. Le seuil de signification de $5 \%$ au test de Tukey a été retenu. 


\section{RESULTATS}

Discrimination des paramètres physico - chimiques et biologiques par la zone sous houppier de Albizia lebbeck et de Gliricidia sepium: L'Analyse en composantes principales (ACP) représente les individus (à gauche) et les variables actives (à droite). Les deux axes expliquent $64,16 \%$ de l'inertie. Les résultats de
l'ACP discriminent les trois types de traitement. En considérant le graphique des individus et le cercle de corrélation on observe le positionnement des sols sous le houppier des deux espèces et du témoin en fonction des paramètres physico-chimiques et biologiques étudiés (Figure 3).

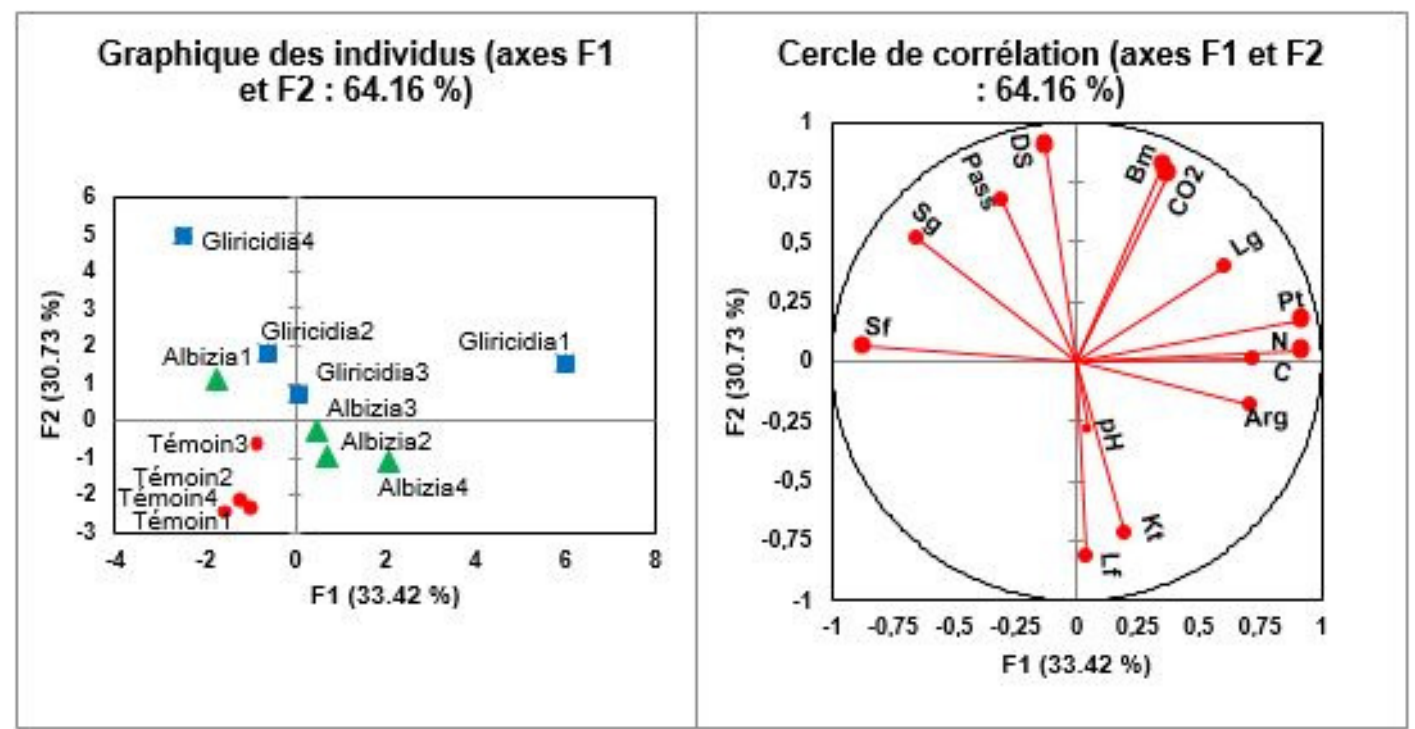

Figure 3 : Discrimination des traitements en fonction des paramètres physico-chimiques et biologiques

Légende : Sg : sable gros, Sf : sable fin, Lg : limon gros, Lf : limon fin, Arg : argile, C : carbone, $\mathrm{N}$ : azote, Kt : potassium total, Pt : phosphore total, Pass : phosphore assimilable, pHe : pHeau, Ds : Densité de spores, Bm : biomasse microbienne, CO2 : activité respiratoire, Point rouge : traitement témoin, Triangle vert : traitement de Albizia lebbeck, Carré bleu : traitement de Gliricidia sepium.

Effets de Albizia lebbeck et de Gliricidia sepium sur la densité de spores et la biomasse microbienne : Le tamisage humide a permis de mettre en évidence la présence de spores des champignons mycorhiziens dans les échantillons de sol collectés. Les résultats consignés dans le tableau 1 montrent que les sols sous Gliricidia sepium comportent une densité de spores (1564,6 $\pm 255,860$ spores/100g de sol) significativement plus élevée $(P=0,013)$ que les sols sous Albizia lebbeck
$(1123,2 \pm 311,239$ spores/100g de sol) et le témoin (889 $\pm 90,288$ spores $/ 100 \mathrm{~g}$ de sol). Les valeurs de biomasse microbienne des sols sous houppier de Gliricidia sepium et de Albizia lebbeck (Tableau 1) sont plus élevées que celles des sols témoins $(P<0,05)$. Ces deux espèces de légumineuses induisent donc une augmentation de la biomasse microbienne des sols sous leurs houppiers.

Tableau 1 : Densité de spores et biomasse microbienne des sols sous houppier de Gliricidia sepium, Albizia lebbeck et des sols témoins.

\begin{tabular}{l|l|l}
\hline Traitement & Densité de spores & Biomasse microbienne \\
\hline Témoin & $889 \pm 90,288 \mathrm{~b}$ & $34,02 \pm 4,524 \mathrm{c}$ \\
Albizia lebbeck & $1123,2 \pm 311,239 \mathrm{~b}$ & $44,4 \pm 4,933 \mathrm{~b}$ \\
Gliricidia sepium & $1564,6 \pm 255,860 \mathrm{a}$ & $61,26 \pm 1,331 \mathrm{a}$
\end{tabular}

Deux valeurs de la même colonne suivies de la même lettre ne diffèrent pas significativement au seuil de $5 \%$ selon le test de Tukey. 
Effets de Albizia lebbeck et de Gliricidia sepium sur l'activité respiratoire: Les courbes de la figure 4 montrent le dégagement de $\mathrm{CO}_{2}$ des sols sous Gliricidia sepium, Albizia lebbeck et le sol témoin. De manière générale, la respiration des sols sous houppier de Gliricidia sepium est plus grande que celle des sols sous houppier de Albizia lebbeck qui à son tour a une respiration plus élevée que les sols témoins.

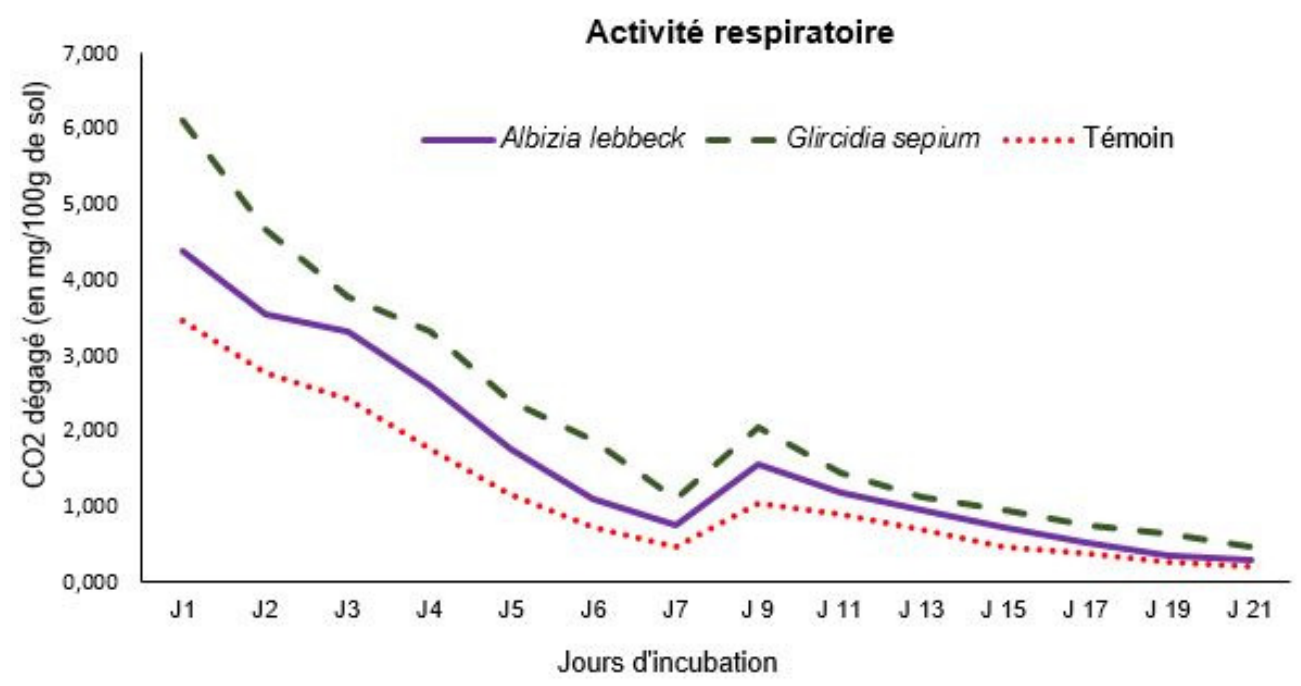

Figure 4 : Evolution de l'activité respiratoire avec le temps d'incubation

La quantité de $\mathrm{CO}_{2}$ dégagée le premier jour par les sols sous Gliricidia sepium, Albizia lebbeck et les sols témoins est respectivement de $6,10 \pm 0,45 ; 4,36 \pm$ 0,55 et $3,44 \pm 0,79$. Au 7 ème jour, elle passe dans le même ordre à $1,08 \pm 0,11 ; 0,74 \pm 0,09$ et $0,44 \pm 0,15$. Le gème jour elle subit une légère hausse avant de

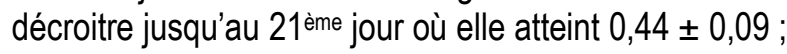
$0,28 \pm 0,04$ et $0,2 \pm 0,001$. Une ANOVA à mesures

\section{DISCUSSION}

Effets de Gliricidia sepium et de Albizia lebbeck sur les paramètres physiques et chimiques du sol: L'analyse en composante principale (ACP) a montré un effet significatif des deux espèces (Gliricidia sepium et Albizia lebbeck) sur les paramètres physico-chimiques et biologiques des sols. En effet, la proportion de ces paramètres du sol est plus élevée dans les sols sous houppier de ces deux espèces. L'effet fertilisant du sol est encore plus important avec Gliricidia sepium qu'avec Albizia lebbeck. Les ligneux ont le potentiel d'influencer quantitativement et qualitativement les paramètres chimiques du sol à travers la litière de leurs feuilles et leurs racines. Concernant l'amélioration de la texture du sol, des auteurs ont trouvé des résultats similaires avec d'autres espèces agroforestières. Yélémou et al., (2013) ont trouvé que la zone sous houppier de Piliostigma reticulatum améliore la texture du sol. Une tendance répétées a été appliquée à l'ensemble des données. L'interaction entre le temps et le type de traitement aurait un effet significatif sur le dégagement de $\mathrm{CO}_{2}$ des sols ( $F[1 ; 2]=28,36 ; p=0,001)$. Cet effet est également grand $\left(\omega^{2}=0,825\right)$. En d'autres termes, la respiration des sols est significativement plus élevée sous Gliricidia sepium suivie des sols sous Albizia lebbeck et des sols témoins.

similaire a également été observée par Ouoba et al., (2018) lors de l'évaluation des potentialités agroforestières de Maranthes polyandra (Benth.) Prance au sud-ouest du Burkina Faso. En effet, la canopée et la litière de Albizia lebbeck et Gliricidia sepium, protègent le sol sous le houppier de l'énergie cinétique de la pluie, ce qui limite le ruissellement et le départ des éléments fins de la surface du sol. Concernant le carbone organique et l'azote (Ndiaye et al., 2012 ; Ouoba et al., 2018) ont montré que les sols sous houppier des espèces de légumineuses telles que Cordyla pinnata et Maranthes polyandra (Benth.) Prance, contenaient plus de carbone et d'azote que les zones hors houppier. Gnahoua et al., (2013) ont également montré que la litière des espèces de légumineuses telles que Acacia auriculiformis, Acacia mangium, Albizia lebbeck et Leucaena leucocephala libère assez d'azote de 
phosphore et de potassium pour le sol. Enfin, Nsombo et al., (2016) ont rapporté des valeurs élevées de phosphore sous la légumineuse Acacia sp. lors de l'évaluation de l'effet de plantations d'Acacia sp sur les macronutriments primaires des sols en République Démocratique du Congo.

Effets de Gliricidia sepium et de Albizia lebbeck sur les paramètres biologiques du sol: Parmi les légumineuses étudiées, Gliricidia sepium est l'espèce qui induit un effet positif important sur la vie du sol. En effet, la densité de spores, la biomasse microbienne et la respiration du sol sont significativement plus importants sous le houppier de Gliricidia sepium que le témoin. Les plantes ligneuses, en améliorant le taux d'humidité, la température et le niveau d'éléments nutritifs ( $N, P$ et $K$ ) dans le sol rendent optimales les conditions de développement des champignons mycorhiziens (Haougui et al., 2013). En outre Meddich et al. (2015) lors de la caractérisation des paramètres physico-chimiques et des potentialités mycorhizogènes des sols salés de la palmeraie Nord-est de Marrakech, ont montré que le nombre des spores de champignons mycorhiziens est affecté par les variations des propriétés physico-chimiques de sols notamment la conductivité électrique, le $\mathrm{pH}$ et les teneurs en phosphore assimilable. Cela confirme que Gliricidia sepium développe des bonnes conditions physico-chimiques $\mathrm{du}$

\section{CONCLUSION ET APPLICATION DES RÉSULTATS}

Cette étude a montré que les deux espèces de légumineuses Albizia lebbeck et Gliricidia sepium ont un effet significatif sur la vie du sol et ses paramètres physico-chimiques. L'activité biologique est dense sous houppier de ces espèces ce qui a pour conséquence l'amélioration de la fertilité du sol. L'espèce Glircidia sepium s'avère encore plus performante pour augmenter la densité des spores du sol, la biomasse sol comme il apparait dans la figure 3. L'effet induit par les légumineuses Gliricidia sepium et Albizia lebbeck sur la vie du sol aurait pu être encore plus significatif si les plantations avaient été plus âgées. En effet, Boureima et al., (2019), sur des régénérations naturelles assistées (RNA), ont montré que la densité des spores dans le sol sous RNA croit en fonction de l'âge des traitements. Concernant l'activité respiratoire, Dabré et al. (2017) ont obtenu des résultats similaires avec des amendements organiques et phosphatés sous zaï. C'est donc dire que l'augmentation de la biomasse microbienne et de l'activité respiratoire du sol induite par les deux espèces étudiées est dû au dépôt de débris organiques (biomasse foliaire et racinaire) sous ces plantes car les débris organiques constituent une source d'énergie pour les microorganismes (Petitjean et al., 2019). Zoghlami et al., (2016) qui ont étudié la minéralisation du carbone et sa corrélation avec la biomasse microbienne ont montré que l'activité respiratoire du sol est fortement corrélée à la biomasse microbienne et en plus elle croit avec l'augmentation du carbone du sol. Ces résultats traduisent également la diversité et l'abondance des micro-organismes sous houppier de ces deux espèces étudiées. En effet la respiration microbienne est considérée comme un indicateur pertinent pour décrire le fonctionnement biologique d'un sol (Montaigne et al., 2018).

microbienne et la respiration du sol. Ces résultats qui auraient pu être plus important si l'âge des plantations était grand, permettent de conclure que l'utilisation de ces espèces en association avec les cultures garantit une exploitation durable des sols. Ces résultats pourraient améliorer l'offre des agriculteurs de la zone soudanienne sur le choix des espèces agroforestières.

\section{REMERCIEMENTS :}

Ce travail a été rendu possible grâce à un appui financier du projet APAF (Association pour la promotion de l'agroforesterie).

\section{RÉFÉRENCES CITÉES}

Bambara D., Bilgo A., Sawadogo J., Gnankambary Z., Thiombiano A., 2018. Évaluation de la diversité et de la qualité de pratiques d'agriculteurs face à la dégradation du milieu biophysique au Burkina Faso. Journal of Applied Biosciences, vol 125(1). 2551-12565.

Blagna F., 2016. Efficacité d'utilisation des phosphates naturels ouest africains par le sorgho ([sorghum bicolor (l.) moench]) associé à des champignons mycorhiziens arbusculaires. Mémoire, Université Joseph KI-ZERBO, 102p.

Boureima S., Ibrahim M., Ibrahim D., et Lawali, S., 2019. Les pratiques paysannes de regénération naturelle assistée des arbustes favorisent le développement des champignons mycorhiziens arbusculaires. Agronomie 
Africaine vol 31, 147 - 158.

BUNASOLS, 2001. Etude morphopedologique de la province de la Sissili et du Ziro/ Rapport technique $n^{\circ} 120.83 p$.

Coulibaly K., Vall E., Autfray P., Nacro HB. \& Sédogo MP., 2012. Effets de la culture permanente coton-maïs sur l'évolution d'indicateurs de fertilité des sols de l'Ouest du Burkina Faso. International Journal of Biological and Chemical Sciences,6, 1069-1080.

Dabré A., Hien E., Somé D. et Drevon JJ., 2017. Effets d'amendements organiques et phosphatés sous zaï sur les propriétés chimiques et biologiques du sol et la qualité de la matière organique en zone soudano-sahélienne du Burkina Faso. International Journal of Biological and Chemical Sciences, vol 11(1). 473-487.

Gnahoua GM., Oliver R., Nguessan KA. \& Balle P. 2013. Production et retombées minérales des litières chez quatre espèces de légumineuses arborées, utilisées en amélioration de jachères, en zone forestière de Côte d'Ivoire. Journal of Applied Biosciences, vol. 72, 5800-5809.

Haougui A., Souniabe PS., Doumma A., et Adam T., 2013. Evolution des populations des champignons endomycorhiziens sur les adventices de quatre sites maraîchers de la région de Maradi au Niger. International Journal of Biological and Chemical Sciences, vol 7, 554-565.

Kouadio K-KH., Ettien DJ-B., Bakayoko S., Soro D. et Girardin O., 2014. Etude de la culture en couloirs de manioc (Manihot esculenta CRANTZ) à base de Gliricidia sepium en Côte d'Ivoire. Afrique SCIENCE. vol 10, 273 - 287.

Meddich A., Hafidi M., Ait El mokhtar M. et Boumezzough A., 2015. Characterization of physicochemical parameters and mycorrhizal potential of salt soils of North-east date palm grove of Marrakesh. J. Mater. Environ. Sci., vol 6 (9). 2469-2475.

Montaigne W., Debon H., Domenach A-M., et Roggy JC., 2018. Gestion durable de la fertilité des sols par l'utilisation de matières organiques : retours d'expérience en Guyane française. Innovations Agronomiques, vol. 64,.71-82.

Nacro S., Ouédraogo S., Traoré K., Sankara E., Kaboré C. \& Ouattara B., 2010. "Effets comparés des pratiques paysannes et des bonnes pratiques agricoles de gestion de la fertilité des sols sur les propriétés des sols et les rendements des cultures dans la zone sud soudanienne du Burkina Faso. International Journal of Biological and Chemical Sciences, vol. 4, 10441055.

Ndiaye Samba SA., Elhadji F., Tala G., Hank M. \& Camire C., 2012. Cordyla pinnata améliore les propriétés du sol et la productivité des cultures. International Journal of Biological and Chemical Science, vol 6, 714-725.

NDT. 2017.Le Concept de Neutralité En Matière de Dégradation Des Terres ( NDT ): Genèse et Définition Réalisation Du Processus Au Burkina Faso. Réseau sahel désertification, Ouagadougou, $5 \mathrm{p}$.

Nsombo MB., Lumbuenamo SR., Aloni KJ., Lejoly J. et Mafuka M-M.P., 2016. Effet des plantations d'Acacia sp sur les macronutriments primaires des sols sableux d'lbi village au plateau des Bateke (Kinshasa, République Démocratique du Congo). Revue Scientifique et Technique Foret et Environnement Du Bassin Du Congo, Vol. 6, 20-27.

Nsombo BM., Lumbuenamo SR., Lejoly J., Aloni JK. et Mafuka PM-M., 2016. Caractéristiques des sols sous savanes et sous forèt naturelle sur le plateau des Batéké en République Démocratique du Congo. TROPICULTURA, vol 34(1). 87-97.

Ouédraogo B., Kaboré O.\& Kaboré M., 2019. Cartographie quantitative de l'érosion des sols par approche SIG/RUSLE dans la Commune de Karangasso vigué (Burkina Faso). International Journal of Biological and Chemical Sciences, vol 13, 1638-1653.

Ouédraogo J., Ouédraogo E. \& Nacro HB., 2014. Effet de l'interaction entre des modes de gestion de fertilité et la macrofaune sur la productivité du niébé et du sorgho en zone nord soudanienne du Burkina Faso. International Journal of Biological and Chemical Sciences, vol 8, 104114.

Ouoba P., Yaméogo JT., Ouédraogo A. et Kouaman S., 2018. Potentialités agroforestières de Maranthes polyandra ( Benth .) Prance au sudouest du Burkina Faso. Journal of Applied Biosciences, vol 128, 12920-12931.

Petitjean C., Philibert A., Manneville V., Amiaud B., Perrin A-S., Charrier X., Gastal F., De Vliegher A., Willekens K., Montenach D., Houot S., Morvan T. et Piutti S., 2019. Biomasse 
microbienne carbonée et activités enzymatiques : gammes de valeurs obtenues pour différents sols agricoles français et belges. Étude et Gestion des Sols, Vol 26, 81-92.

Ramdé-Tiendrebéogo A., Zerbo R., Ouattara B., Doulkom A. et Guissou IP., 2019. Plantes sahéliennes adaptées dans la récupération des terres dégradées et leurs usages pour la santé : cas de la province du Soum au Nord du Burkina Faso. Journal of Animal \& Plant Sciences, vol. 41, 6767-6783.

REEB IV., 2017. Quatrieme rapport sur l'état de l'environnement au Burkina Faso. SP/CNDD, Ouagadougou, 271p.

Savadogo PW., Zi Y., Sanou AK., Nacro HB., Lompo F. et Sédogo MP., 2017. Effets combinés du compost, du Paraquat et de la Lambdacyhalothrine sur la macrofaune du sol sous culture pluviale de sésame (Sesamum indicum L.) au Burkina Faso. International Journal of Biological and Chemical Sciences, vol. 11, 2658-2670.

Somé D., Hien E., Assigbetse K., Drevon JJ. et Massé D., 2015. Dynamique des compartiments du carbone et de l'azote dans le sol cultivé en niébé et sorgho dans le système zaï en zone Nord soudanienne du Burkina Faso. International Journal of Biological and Chemical Sciences, vol. 9, 954-969.

Villenave C., Chauvin C., Santune C., Cérémonie H. et Schneider A., 2018. L'effet des légumineuses sur le fonctionnement biologique du sol : une méta-analyse sur la nématofaune du sol. Innovations Agronomiques, vol. 69, 47-60.

Yaméogo JT., Sanon Z., Baggnian I., Somda I., Somé AN. et Axelsen JA., 2019. Impact des différents types d'occupation des terres sur la fertilité physique et chimique du sol dans la réserve totale et partielle de Bontioli (Sud-Ouest) du Burkina Faso. Science et technique, Sciences naturelles et appliquées, vol. 38, 33-45.

Yélémou B., Yaméogo G., Barro A., Taonda SJ., et Hien V., 2013. La production de sorgho dans un parc à Piliostigma reticulatum en zone nordsoudanienne du Burkina Faso. TROPICULTURA, vol. 31, 154-162.

Zoghlami, R. I., Aissa, N. B. E. N., Hamdi, H., \& Khelil, M. N. (2016). Minéralisation du carbone organique et sa corrélation avec la biomasse microbienne dans deux sols agricoles suite à un amendement de boues résiduaires. 31(11),
1812-1821.

Zongo CB., 2013. Etude Diagnostique de La Production Du Compost Dans La Province Du Yatenga. Mémoire de fin de cycle, IDR, Bobo-Dioulasso (Burkina Faso), 72p. 